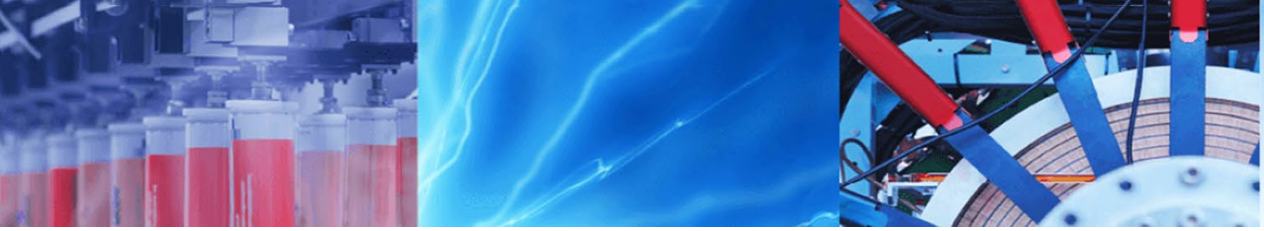

Research Article

\title{
Isolation and characterization of ginseng-derived exosome-like nanoparticles with sucrose cushioning followed by ultracentrifugation
}

\author{
Jisu Kim ${ }^{1}$ Y Yeon-Hee Lee ${ }^{2}$ (D) Jianxin Wang ${ }^{1,3} \cdot$ Youn Kyung Kim² ${ }^{2}$ II Keun Kwon ${ }^{4}$
}

Received: 23 July 2021 / Accepted: 11 January 2022

Published online: 31 January 2022

(c) The Author(s) 2022 OPEN

\begin{abstract}
Ginseng is a traditional medicine that has been used for millennia. Asian ginseng (Panax ginseng Meyer) has multiple pharmacological properties. To determine the efficacy of ginseng-derived exosome-like nanoparticles (GDEs), GDEs should be isolated and characterized. GDEs from $P$. ginseng were isolated via sequential centrifugation using 68 and $27 \%$ of sucrose cushioning followed by ultracentrifugation. The size distribution, zeta potential, and morphology of GDEs were evaluated using DLS, NTA, and TEM. GDEs were quantified by measuring the total RNA and protein concentration. The stability of the freeze-dried GDEs was evaluated for 90 days. Uniform and reproducible GDEs were successfully isolated with high yield and purity. GDEs were found to be spherical with an average diameter of $105.8 \mathrm{~nm}$ and a negative surface charge of $-20.7 \mathrm{mV}$. Substantial amounts of nanoparticles of GDEs were counted using NTA, with a concentration of $2.05 \times 10^{13}$ particles $/ \mathrm{mL}$. According to the evaluation of their protein and total RNA concentration, the freeze-dried GDEs were stable for up to 60 days at room temperature. The results suggest the utility of a brief isolation protocol using sucrose double fractionation, which can be used for the mass production of plant-derived exosome-like nanoparticles. In addition, the stability of GDEs was maintained even after freeze-drying and storage for 60 days. This method has potential for application in the research for plant-derived exosome-like nanoparticles, where cost-effective exosome isolation with high yield and purity is an important step.
\end{abstract}

Keywords Ginseng · Exosome · Plant-derived exosome-like nanoparticle · Ultracentrifugation · Nanovesicles · Isolation

\section{Abbreviations}

DLS Dynamic light scattering

DNA Deoxyribonucleic acid

EV Extracellular vesicles

GDEs Ginseng-derived exosome-like nanoparticles

NTA Nanoparticle tracking analysis

RNA Ribonucleic acid

TEM Transmission electron microscopy

\section{Introduction}

Asian ginseng (Panax ginseng Meyer) is known as the king of herbs. Ginseng has multiple pharmacological properties in cardiovascular diseases, diabetes, and cancer, and has been used to promote immune function, central nervous system function, relieve stress, and enhance bone remodeling [1]. It has been used in traditional medicine for the treatment of diseases in East

Yeon-Hee Lee and Jianxin Wang have contributed equally.

\footnotetext{
$\triangle$ Yeon-Hee Lee, omod0209@gmail.com; $\bowtie$ Jianxin Wang, jxwang@fudan.edu.cn| ${ }^{1}$ Department of Pharmaceutics, School of Pharmacy, Fudan University \& Key Laboratory of Smart Drug Delivery, Ministry of Education, Shanghai 201203 , China. ${ }^{2}$ Department of Orofacial Pain and Oral Medicine, Kyung Hee University Dental Hospital, \#26 Kyunghee-daero, Dongdaemun-gu, Seoul 02447, Korea. ${ }^{3}$ Institute of Integrated Chinese and Western Medicine, Fudan University, Shanghai 200040, China. ${ }^{4}$ Department of Dental Materials, School of Dentistry, Kyung Hee University, Seoul 02447, Korea.
}

SN Applied Sciences $\quad$ (2022) 4:63 $\quad$ https://doi.org/10.1007/s42452-022-04943-y 
Asian countries for millennia, and until recently, it has become one of the most effective herbs ${ }^{2}$. Moreover, ginseng has been investigated for its therapeutic potential in stress, hypertension, osteoporosis, and various neurological disorders such as Parkinson's disease and Alzheimer's disease [3, 4] (Fig. 1). Although numerous molecular targets for ginseng have been identified in recent years $[5,6]$, it is still not clear which factors are responsible for the therapeutic effects of ginseng.

Exosomes are small membrane vesicles $(50-150 \mathrm{~nm})$ of endocytic origin and are produced under normal as well as pathological conditions. Generally, exosomes can be produced by almost every living cell, and have been isolated from archaea, prokaryotic cells, eukaryotic cells, and plant tissues. Exosomes were thought to function only as "trash cans" until 20 years ago [7]. However, recently, exosomes have been considered as key mediators of cell-to-cell communication task to control both normal physiological processes and disease pathophysiology [8]. The content profiles of exosomes are mainly determined by the parent cell and the environmental conditions in which they originate, and affecting surrounding cells [9]. Additionally, exosomes also have intrinsic biological activity and are capable of carrying unique surface receptors and therapeutic cargo including proteins, lipids, RNAs, and DNA [10]. Moreover, several studies have demonstrated that exosomes can be used as therapeutic or drug delivery platforms owing to their nanoscale size, low toxicity, excellent structural stability, and cargo loading ability [11, 12].
In plants, consensus among researchers regarding specific markers for extracellular vesicle subtypes, such as the exosomes of endosomal origin in animal cells, has not been reached. Therefore, it is difficult to assign the widely accepted term "exosome" to plant-derived extracellular nanovesicles, and accordingly, the term "exosome-like nanoparticles" has been used herein $[13,14]$. Plant-derived exosome-like nanoparticles have become a new area of interest owing to their remarkable advantages, such as non-toxicity, biocompatibility, and stability [15]. Exosome-like nanoparticles isolated from fruits and plants have been explored for clinical use because they come from reliable sources and have better safety profiles $[16,17]$. In particular, ginseng-derived exosome-like nanovesicles have been demonstrated to have many therapeutic effects, including anti-cancer, anti-inflammatory, antioxidant, and neuroprotective properties [18]. In addition, compared to animal cell culture media, plants are a more favorable source for increasing the overall exosome yield [19].

However, there is still a lack of comprehensive studies focusing on the characterization of plant-derived extracellular vesicles, practical quantitative analysis of their characteristics, and methods for their mass production. Exosomes isolated from mammalian cells and tissues have been characterized for their size, morphology, concentration, and surface marker protein expression [20]. Most studies have been on mammalian cell-derived exosomes, and human knowledge about therapeutic cargo on plant-derived

Fig. 1 Biological therapeutic activity of Panax ginseng

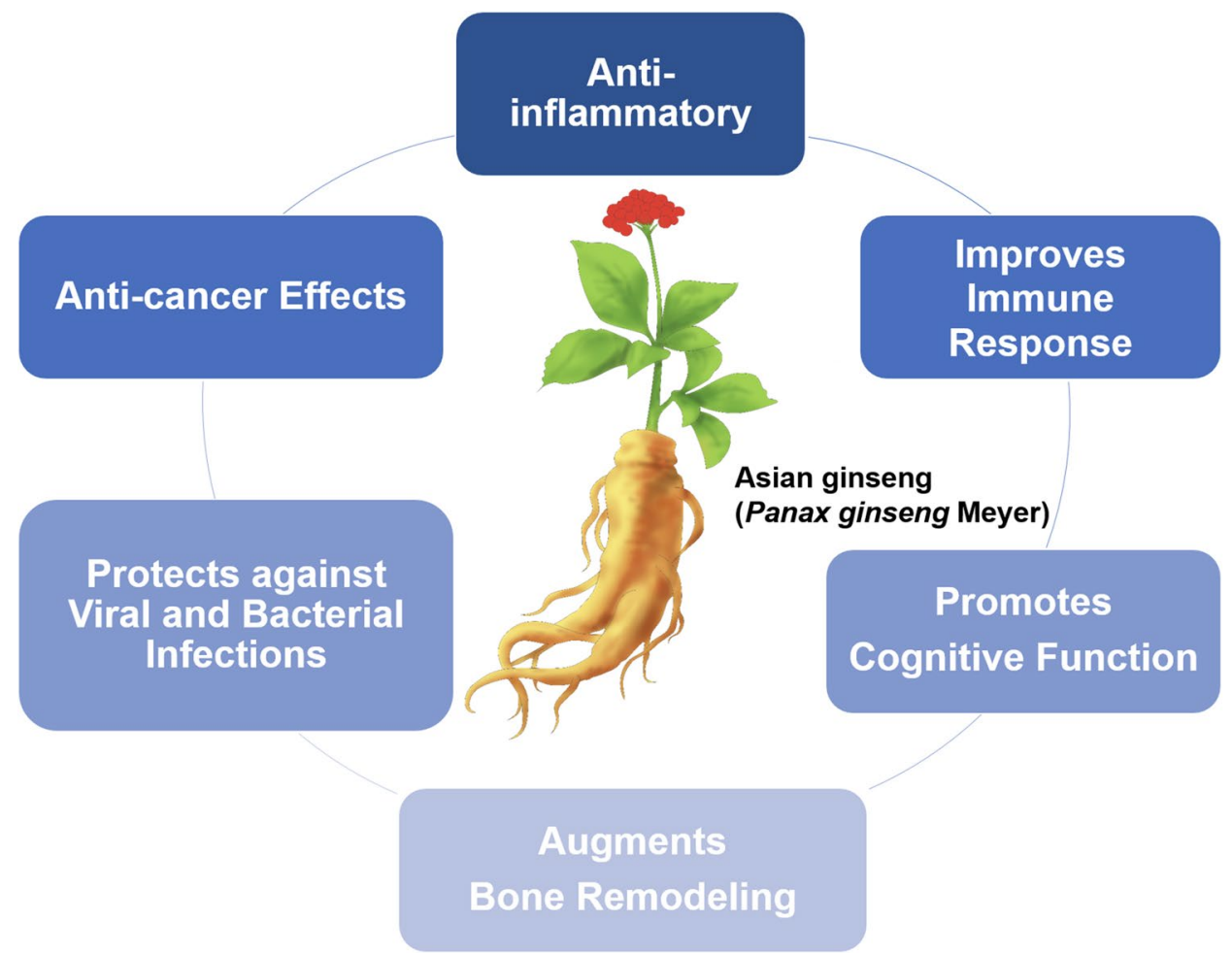

SN Applied Sciences 
exosome-like nanoparticles is relatively scarce. Although several strategies have been developed to scale up gingerderived exosome like nanoparticles production, economically, the cost/yield ratio is still not favorable for clinical application [21].

Thus, in this study, we demonstrate the isolation of plant exosome-like nanovesicles from ginseng using a newly developed double fractionation ultracentrifugation method and evaluated their characteristics such as size, surface charge, and physical morphology before and after freeze-drying. We evaluated whether a novel sucrose cushioning method and sequential centrifugation could be used to obtain homogeneous and reproducible ginseng-derived exosome-like nanoparticles (GDEs) with high yield and purity. Furthermore, we performed a quantitative analysis by measuring the protein and total RNA concentration of GDEs and assessed their stability for 90 days. Here, we present an effective and economical GDE isolation protocol for the potential use of GDEs in clinical and experimental applications.

\section{Results}

\subsection{Isolation and characterization of GDEs}

Using a modified centrifugation-based isolation method with an additional sucrose cushion, we isolated GDEs from $P$. ginseng. After sequential low-velocity centrifugation steps, large impurities and debris were discarded (Fig. 2b). To protect their round-shaped morphology and prevent them from being contaminated with proteins, we utilized a sucrose cushion to purify ginseng extracellular vesicles (EVs). Sucrose solutions (68\% and $27 \%$ solutions) were placed at the bottom of the polyethylene tube to prevent disturbing the interface, and the supernatant was gently transferred to the top of the sucrose layers. After centrifugation at a high velocity, the ginseng EVs were purified between the sucrose cushion layers (Fig. 2c). The ginseng EVs were collected and placed on top of the sucrose gradient solution. Furthermore, the GDEs were subjected to ultracentrifugation and isolated between the $8 \%$ and $30 \%$

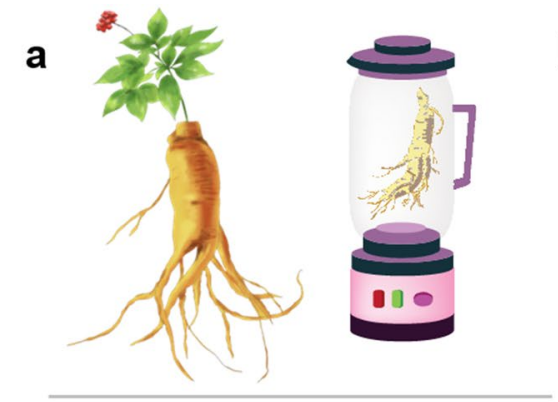

I . Grinding fresh ginseng

Preparation of ginseng

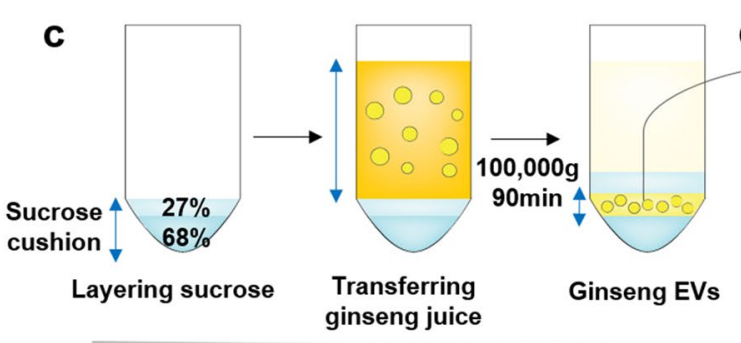

III. Ultracentrifugation

b

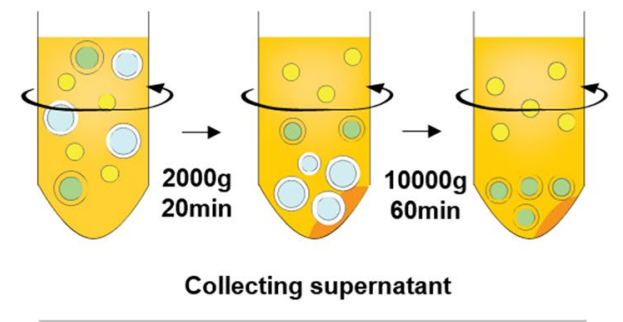

II. Medium to high velocity centrifugation

Purification of ginseng juice

d Ginseng EVs

d
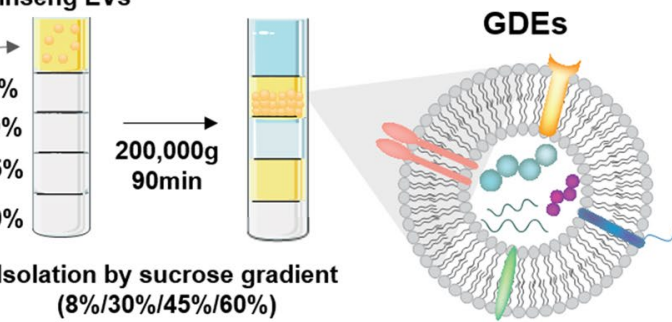

Isolation of ginseng-derived exosome like nanoparticles

Fig. 2 Isolation of GDEs. a a 4-year-old Panax ginseng was obtained and put into a blender containing $1 \times$ phosphate-buffered saline (PBS). b the ginseng juice was sequentially centrifuged at low velocity to remove debris. c sucrose cushion solutions at $68 \%$ and $27 \%$ were slowly added to ultracentrifuge polycarbonate bottles without mixing the two layers, and the supernatant was gently transferred to the bottle. After ultracentrifugation, the nanovesicles were collected between the sucrose cushions. $\mathbf{d}$ to isolate GDEs, the nanovesicles were purified using a sucrose gradient $(8 / 30 / 45 / 60 \%)$. GDEs were obtained between the $8 \%$ and $30 \%$ sucrose layers 
sucrose solutions (1.13-1.19 g/mL concentration) (Figs. $2 \mathrm{~d}$ and 3a). To characterize GDEs, via particle-by-particle investigation, the size distribution and particle concentration were measured via Nano-tracking analysis (NTA). As shown in Fig. 3b, NTA indicated that GDEs harvested through the modified sucrose cushion method appeared to be homogeneous vesicles and had a concentration of approximately $2.05 \times 10^{13}$ particles $/ \mathrm{mL}$. We also performed transmission electron microscopy (TEM) imaging to investigate the morphology of GDEs. The results showed that GDEs retained a uniform spherical shape with no detectable aggregation due to protein contamination. Dynamic light scattering (DLS) measurements based on number confirmed that the average diameter of GDEs was approximately $105.8 \pm 47.85 \mathrm{~nm}$ with a low polydispersity index (PDI) at 0.257. GDEs had a negative surface charge of $-20.7 \mathrm{mV}$ (Fig. 3d). Overall, the performance of the modified isolation method with the sucrose cushion for the isolation of GDEs enabled the generation of reproducibly uniform GDEs due to the reduced degradation of ginseng EVs after repeated exposure to strong centrifugal forces.

Furthermore, to accurately determine the diameter of GDEs, a technique based on the intensity, volume, and number of GDEs using scattered light was applied in DLS (Fig. 4). The diameter and size distribution can be detected through fluctuations caused by Brownian motion in the hydrodynamic volume of the nanoparticles. However, the fluctuations in the scattered light may be affected by particle size, size polydispersity, and shape of the nanoparticles; thus, the nanoparticles could be determined differently from real objects. Consequently, the size distribution of GDEs based on their number, best represented the realistic size of GDEs and exhibited a homogeneous particle size, as observed by TEM imaging (Fig. 4c). In contrast, temporary aggregates or large particles of GDEs detected in size distribution profiles were based on the intensity and volume. DLS is highly sensitive for measuring the size distribution of GDEs and is considered appropriate for determining the size of GDEs. The actual size of GDEs was markedly similar between DLS measurements based on number and microscopic observation.

\subsection{Characterization of freeze-dried GDEs}

GDEs isolated using the sucrose cushion method were lyophilized to determine the possibility of the long-term preservation of GDEs and their components. After isolation, the liquid phase of GDEs was frozen at $-80^{\circ} \mathrm{C}$ for $1 \mathrm{~h}$, transferred to a freeze-drying chamber, and lyophilized using a freeze dryer under a pressure of $0.072 \mathrm{mbar}$ at $-87.5^{\circ} \mathrm{C}$ for $72 \mathrm{~h}$. The aqueous solution of GDEs was rapidly converted
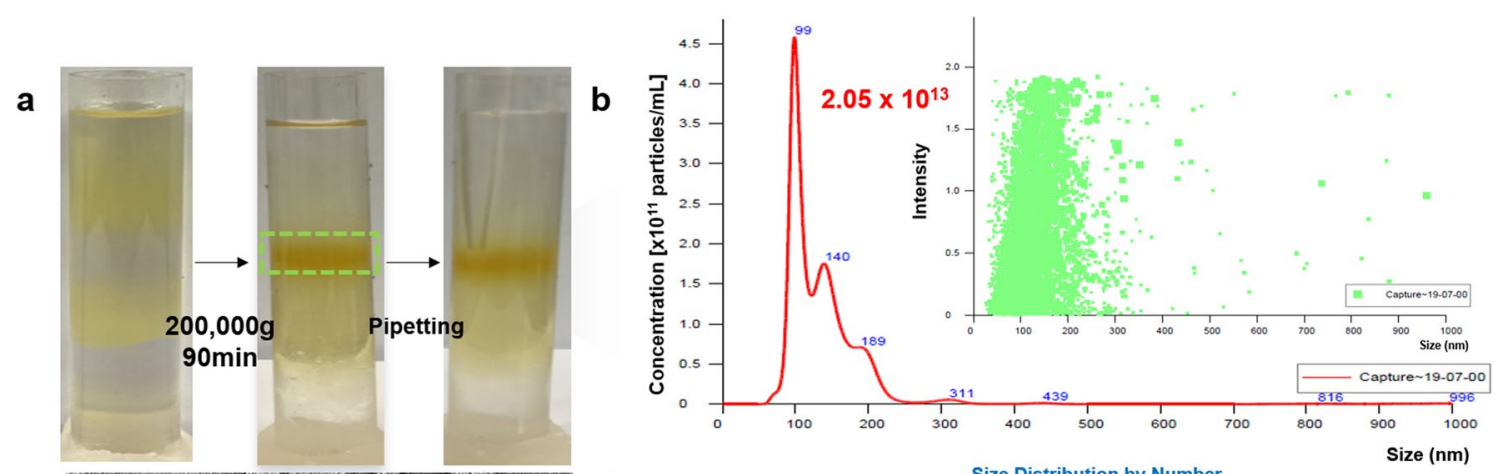

C

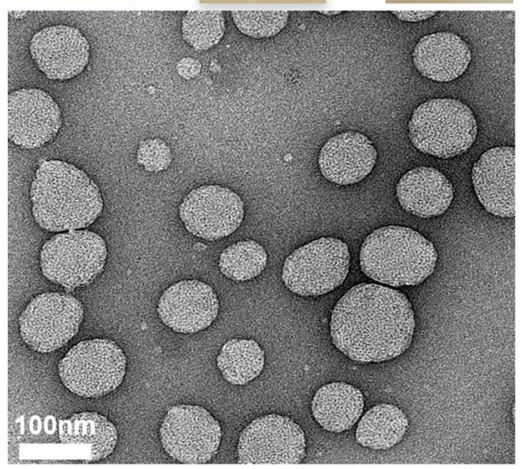

d
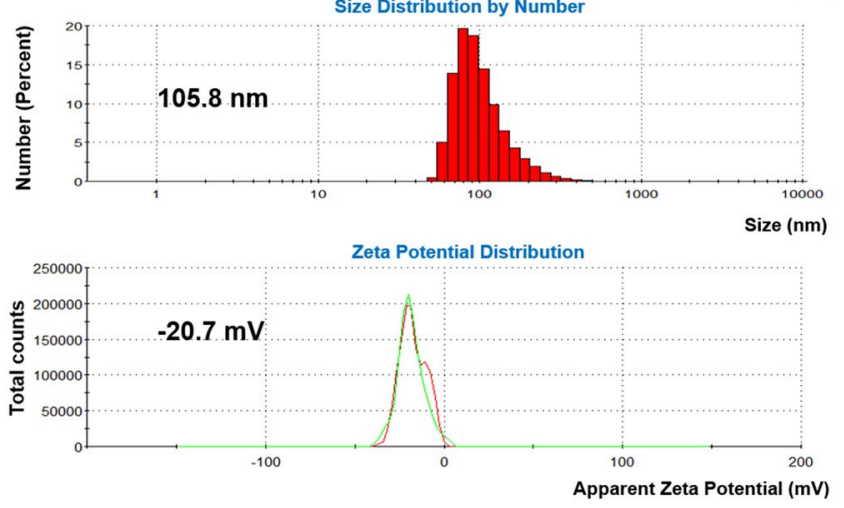

Fig. 3 Characterization of ginseng-derived exosome-like nanoparticles (GDEs). a Isolation of GDEs. b nanoparticle tracking analysis results showing the size distribution and number of GDEs. $\mathbf{c}$ trans-

mission electron microscopy image of GDEs. $\mathbf{d}$ dynamic light scattering analysis results showing the size and zeta potential of GDEs 
Fig. 4 Measurement of the diameter of GDEs based on size distribution using the dynamic light scattering technique. a-c measurement of size distribution of GDEs by intensity, volume, and number. d particle size distribution by intensity, volume, and number and the standard and mean deviation of the DLS peaks a

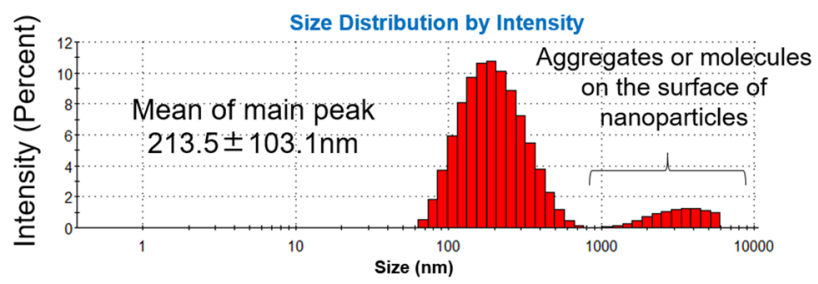

b

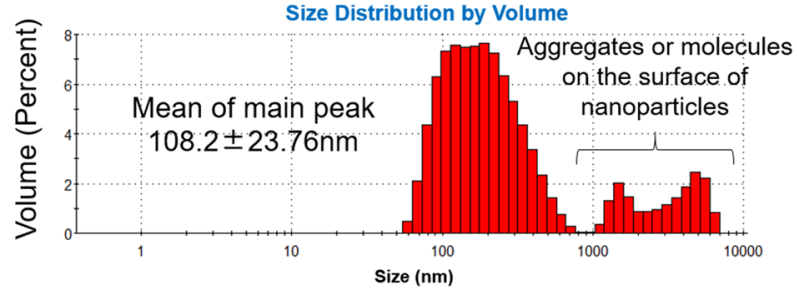

c

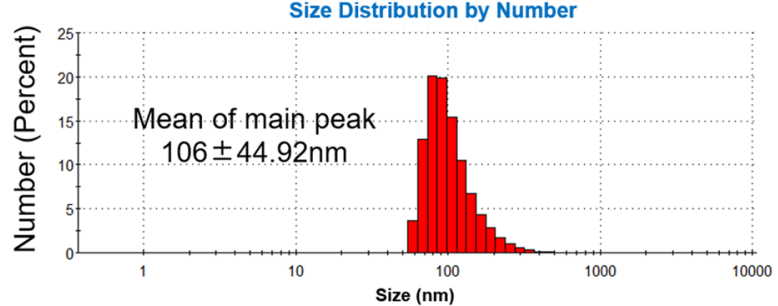

d

\begin{tabular}{ccccccc}
\hline & \multicolumn{2}{c}{ Peak 1 } & \multicolumn{2}{c}{ Peak 2 } & \multicolumn{2}{c}{ Peak 3 } \\
\hline & Average $(\mathrm{nm})$ & $\%$ & Average $(\mathrm{nm})$ & $\%$ & Average $(\mathrm{nm})$ & $\%$ \\
Intensity & $213.5 \pm 103.1$ & 90.7 & $3408 \pm 1221$ & 9.3 & - & - \\
Volume & $108.2 \pm 23.76$ & 32.8 & $257.3 \pm 114.7$ & 49.9 & $1637 \pm 347.9$ & 6.4 \\
Number & $106 \pm 44.92$ & 100 & - & - & - & -
\end{tabular}

into a powder form through the sublimation and evaporation of water during drying.

To verify the difference between the liquid-phase and freeze-dried GDEs, the sizes and morphologies of the particles were determined using DLS, TEM, and NTA (Fig. 5). We found no detectable changes in the physical properties and integrity of the GDEs before and after freeze-drying. The liquid-phase and freeze-dried GDEs had average diameters of approximately 98.24 and $92.62 \mathrm{~nm}$, respectively. The PDI values of liquid-phase and freeze-dried GDEs were low at 0.172 and 0.267 , respectively. The surface charge indicated that liquid-phase and freeze-dried GDEs had negative zeta potentials, with average charge values of -24.4 and $-26.4 \mathrm{mV}$, respectively (Fig. 5a). Further characterization of liquid-phase and freeze-dried GDEs, as imaged using TEM, revealed that they were generally spherical in shape and evenly spaced, showing a uniform size distribution without damaged particles (scale bar: $100 \mathrm{~nm}$ ) (Fig. 5b). The concentration and dispersion of liquid-phase and freeze-dried GDEs were measured using NTA. There was no damage or loss of particles in freezedried GDEs compared with liquid-phase GDEs (Fig. 5c).

\subsection{Quantitative analysis of GDEs and their stability}

Total RNA was extracted from ginseng tissue and GDEs and characterized based on the length of the isolated RNA by loading it onto a 10\% $8 \mathrm{M}$ urea-denaturing gel (Fig. 6a). In addition, we treated the total RNA with ribonuclease $\mathrm{H}$ (RNase $\mathrm{H}$ ) for $2 \mathrm{~h}$ at $37^{\circ} \mathrm{C}$ and observed the existence of several small-sized exosomal RNAs in GDEs (Fig. 6b). The experimental evidence of the total RNA content in GDEs indicated the presence of genetic information that can be used to predict target genes for various diseases and that these components could be effectively used for the quantitative analysis of GDEs. According to a previous study, the purity of exosomes can be diminished by the isolation process or protein contamination [22]. In practice, purity is considered a crucial factor for the evaluation of the biological activity and availability of exosomes. To evaluate the purity of GDEs, the ratio of particle counts to protein concentration was calculated, and it was observed to be approximately $1.03 \pm 0.11 \times 10^{13}$ particles $/ \mathrm{mg}$ of protein (Fig. 6c). 


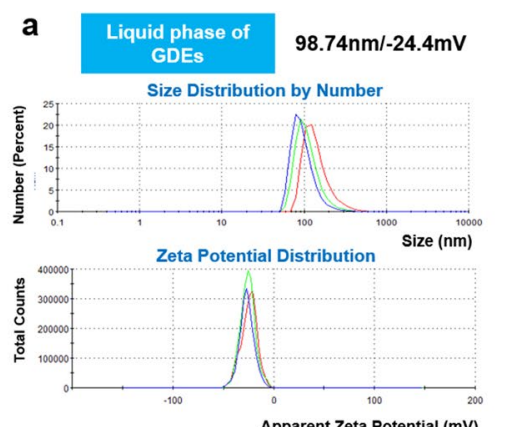

b
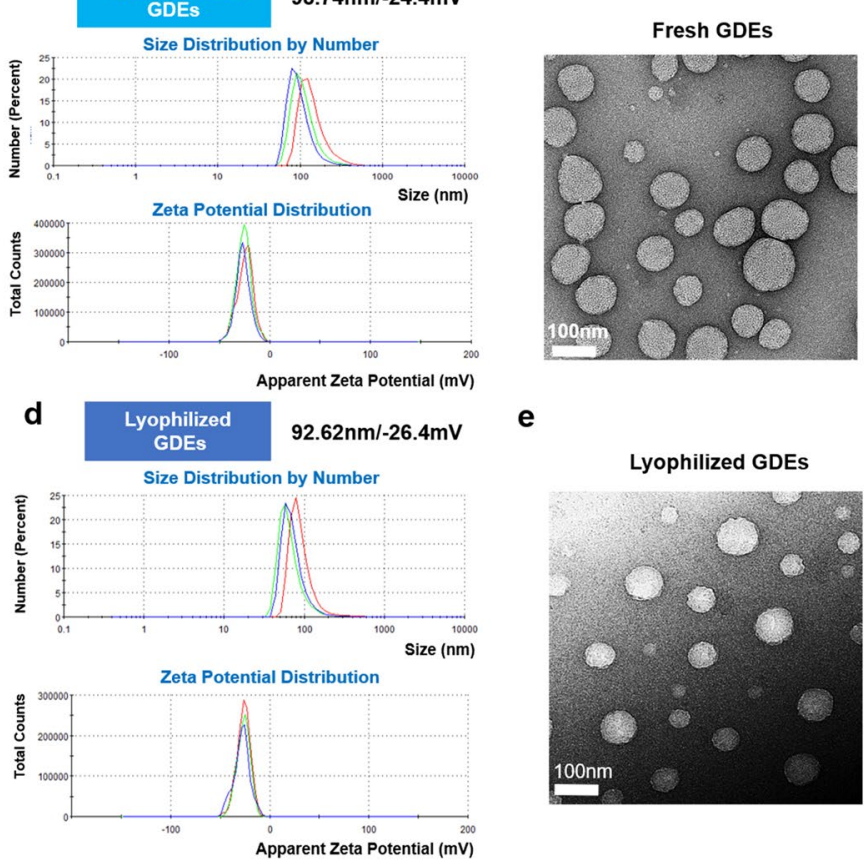

e

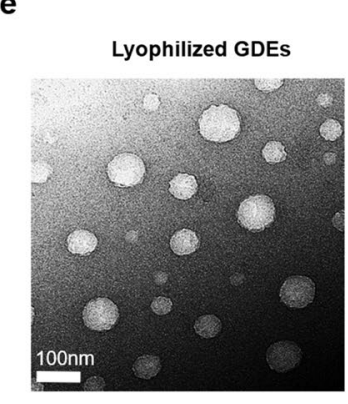

c
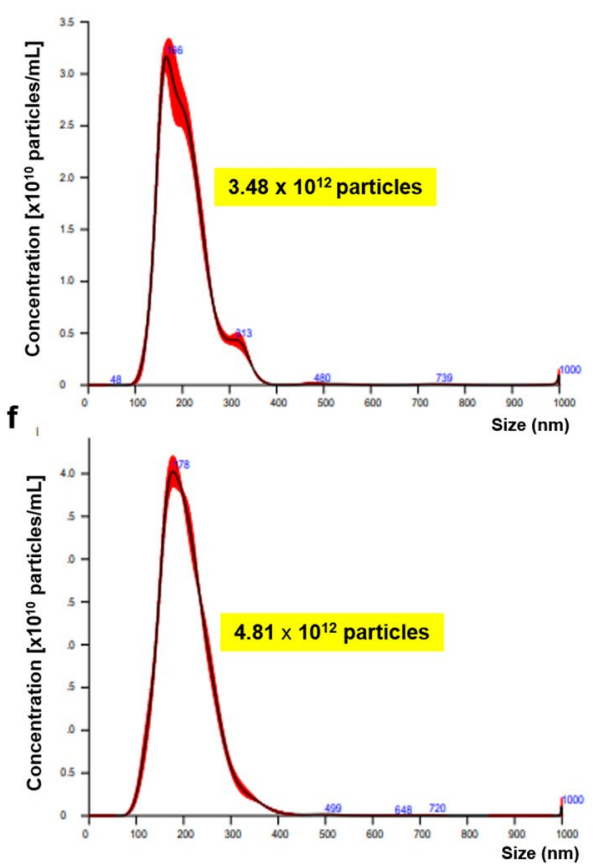

Fig. 5 Characterization of GDEs before and after freeze-drying. a, d DLS measurement of GDEs before and after freeze drying, respectively. b, e representative TEM images of fresh and freeze-fried

GDEs, respectively. c, f size distribution and particle concentration of GDEs as measured via NTA

\section{Discussion}

The yield of GDEs was quantified based on the protein concentration and the total RNA concentration. After freeze-drying, $1 \mathrm{~g}$ of GDEs was weighed and dissolved in $1 \mathrm{~mL}$ of $1 \times$ PBS. The protein concentration of GDEs measured via the Bradford assay was approximately $2.004 \pm 0.22 \mathrm{mg} / \mathrm{mL}$. Total RNA was extracted from $1 \mathrm{~g}$ of freeze-dried GDEs following the TRIzol RNA isolation protocol, and the total RNA concentration was measured to be approximately $51.525 \pm 0.492 \mathrm{ng} / \mu \mathrm{L}$ (Fig. $6 \mathrm{~d}$ ). The yield of GDEs per $10 \mathrm{~g}$ of ginseng tissue was also quantitatively evaluated, with a protein concentration of $4.811 \pm 0.529 \mathrm{mg} / \mathrm{mL}$ and a total RNA concentration of $123.66 \pm 1.182 \mathrm{ng} / \mu \mathrm{L}$ (Fig. 6e).

Furthermore, to determine the stability of the GDEs, freeze-dried GDEs were divided into tubes and stored at room temperature for up to 90 days. The protein and total RNA concentrations of these GDEs were measured during this period (Fig. 7a). Quantitative analysis of the freeze-dried GDEs indicated that they had no significant decreases in protein concentration and total RNA concentration until day 60 . However, they showed a steep decrease in total RNA concentration at day $90 \mathrm{com}-$ pared to day $1(50.88 \pm 1.34 \mathrm{ng} / \mu \mathrm{L}$ to $24.08 \pm 0.07 \mathrm{ng} / \mu \mathrm{L}$, $p<0.001$ ), while their protein content was maintained during the 60-day storage (Fig. 7b).

In this study, we suggested a strategy to isolate exosome-like nanoparticles from Asian ginseng and a protocol so that these vesicles can be economically massproduced. Here, GDEs were purified from ginseng bodies and roots via sequential centrifugation and ultracentrifugation using double fractionation. Ultracentrifugation is the most widely used exosome purification method and has long been regarded as the gold standard for isolating exosomes of relatively homogeneous sizes [23]. Although ultracentrifugation is a powerful tool for optimizing exosome isolation, it is unclear what concentration methods are most appropriate for exosome isolation [24]. The disadvantages of ultracentrifugation include the possibility of sample loss depending on the skill of the operator and that repeated ultracentrifugation steps can damage vesicles and reduce yields, thereby potentially impacting the protein and RNA content of the isolated exosomes [23, 25].

The most remarkable strength of ultracentrifugation is that particle yields can be dramatically higher in centrifuge-concentrating devices than in pressure-driven concentrators. Another advantage of ultracentrifugation is that a concentration gradient can be used to remove contaminating impurities, such as non-specific proteins 
Fig. 6 Total RNA extracted from ginseng tissue and GDEs, quantitative analysis of GDEs, and the effects of storage on these parameters. a total RNA extracted from ginseng tissue and GDEs was separated using b total RNA of GDEs treated with ribonuclease $\mathrm{H}$ (RNase $\mathrm{H}$ ). c purity of GDEs calculated as the number of nanoparticles per protein concentration (mg). d total protein concentration of GDEs determined via a Bradford assay. One gram of GDEs was used to measure the total protein (left) and RNA concentration (right). d the protein and total RNA concentrations converted into GDEs per $10 \mathrm{~g}$ of ginseng tissue. The protein concentration of the GDEs was measured using a Bradford assay (left) and the total RNA concentraNanoDrop (right) a 10\% $8 \mathrm{M}$ urea denaturing gel. tion of GDEs was measured via
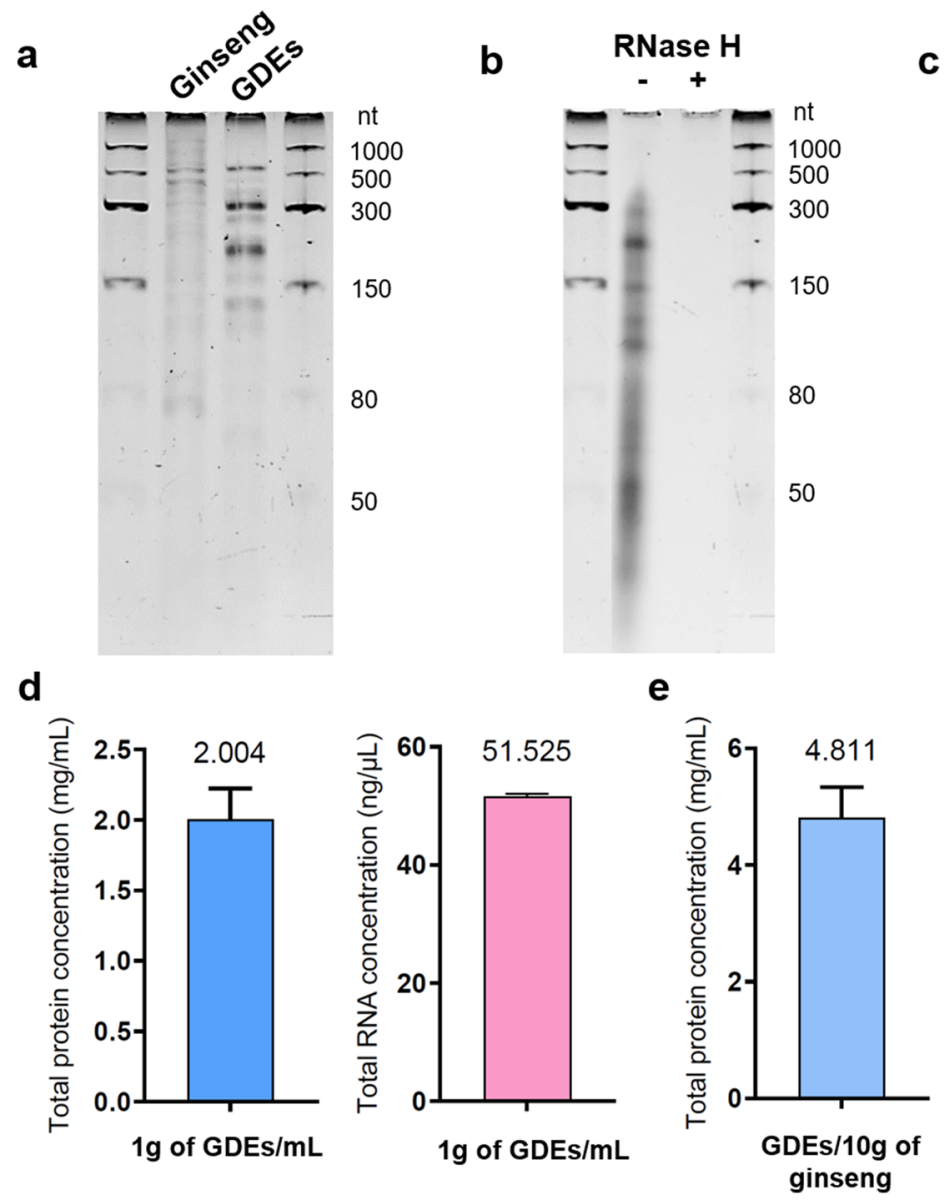

C

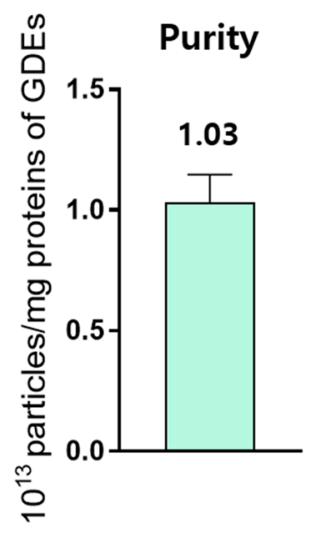

Fig. 7 Evaluation of GDE stability by measuring their total RNA and protein content for 90 days. a the total RNA and protein concentration of GDEs stored at room temperature measured for 90 days after isolation. $\mathbf{b}$ tabular form of the total RNA and protein concentration values in $\mathbf{a}$ a

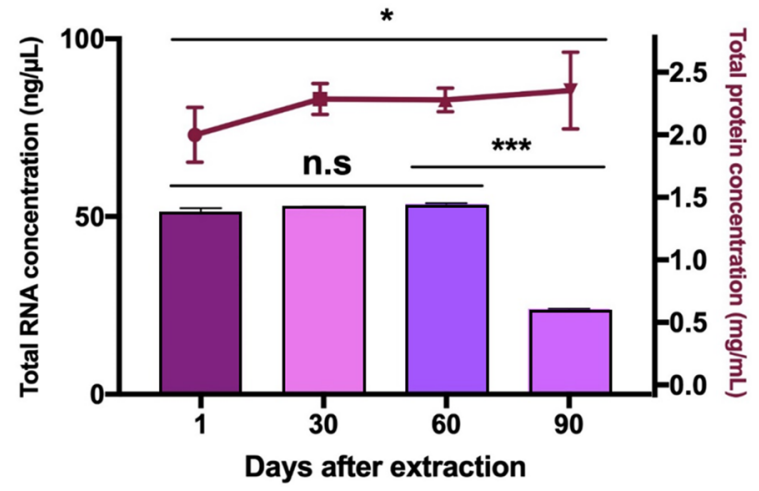

b

\begin{tabular}{ccccc}
\hline Time (Day) & $\mathbf{1}$ & $\mathbf{3 0}$ & $\mathbf{6 0}$ & $\mathbf{9 0}$ \\
\hline $\begin{array}{c}\text { Total RNA (ng/uL) } \\
\text { Total protein } \\
(\mathrm{mg} / \mathrm{mL})\end{array}$ & $50.88 \pm 1.34$ & $52.65 \pm 0.15$ & $53.55 \pm 0.21$ & $24.08 \pm 0.07$ \\
\hline
\end{tabular}

[26]. Our centrifugation protocol achieved a high yield of GDEs with high purity.

We showed that our double purification and fractionation protocol based on the concentration of EVs is capable of isolating exosomes-like nanoparticles and provides a higher particle yield, thereby increasing exosome yield and isolation efficiency. The increased yield in particle concentration is also coupled with a shorter processing time, thereby increasing the efficiency of the sample throughput. The isolation of pure exosomes from 
ultracentrifuged crude exosome pellets from human plasma could be improved via sucrose gradient fractionation [27, 28]. A one-step sucrose cushion ultracentrifugation method was demonstrated to be effective in isolating exosomes from mesenchymal stem cells because of the high yield and purity of the isolates [29]. In a previous study, ginseng-derived exosome-like nanoparticles were isolated through ultracentrifugation with $15 \%, 30 \%, 45 \%$, and $60 \%$ of sucrose gradient using a pellet EVs. [30]. After then, a method for isolating exosomes-like nanoparticles using a sucrose cushion has been proposed, and a unique feature of our study was the application of a double purification method based on concentration using a sucrose cushion and a sucrose gradient separation step before two ultracentrifugation steps followed by two centrifugation steps. Only the advantages have been described without mentioning the disadvantages of this cushioned-density gradient ultracentrifugation [30,31]. Additional research is needed to optimize this method regarding the differences in sucrose concentration, number of sucrose layers, speed and duration of ultracentrifugation, and ginseng type, etc.

In comparison with a previous isolation method of plant-derived exosome-like nanoparticles from carrot, apple, lemon, grape, grapefruits, tea leaves, cabbage, ginger, and garlic [32-38], we established the double fractionation of GDEs by sucrose-based isolation to purify GDEs from vesicle aggregates or contaminated proteins for reproducible high-quality exosome-like nanoparticles. To collect homogeneously sized GDEs with high yield, we first isolated non-contaminated intact EVs from ginseng juice through sucrose cushion of different densities, which can prevent the degradation of EVs during repeated highforce centrifugations. After ultracentrifugation, ginseng EVs obtained were heterogeneous and contained diverse subtypes of EVs. Further fractionation with sucrose gradient solutions ( $8 \%, 30 \%, 45 \%$, and $60 \%$ ) was employed to isolate GDEs from ginseng EVs between the $8 \%$ and $30 \%$ sucrose layers. Eventually, through the double fractionation of GDEs, uniformly round GDEs were obtained. This optimized isolation method can lead to the refinement of exosome-like nanoparticles with high yield and purity, which may have a strong potential in nanomedicine and drug delivery systems.

To date, three subtypes of isolation methods for plantderived exosome-like nanoparticles have been reported: simple sequential ultracentrifugation and pelleting [32], density gradient separation [21,34, 39], and purification with ultrafiltration [37]. According to previously described protocols for isolating plant-derived exosome-like nanoparticles, these methods differ from ours in terms of how to make the cushion in detail, the number of applications, the concentration of sucrose, and the materials and plants used. Furthermore, Cao et. al. obtained ginseng-derived exosome-like nanoparticles isolated via sucrose density gradient separation after pelleting EVs, and the ginsengderived exosome-like nanoparticles they isolated showed comparatively different physical and molecular parameters in terms of particle size, morphological shape, and nanoparticle concentration due to the different isolation processes involved [30]. This was attributed to the damage and degradation of the nanoparticles during their highspeed centrifugation into pellets, so we applied the cushion method in this study to reduce such damage.

GDEs obtained from this brief isolation protocol were characterized through various experimental techniques based on size profiling, surface charge, and protein and RNA concentration. The particle size and distribution the GDEs were measured using DLS and TEM, and it was observed that GDEs had a spherical shape with an average diameter of $105.8 \mathrm{~nm}$ and a negative surface charge of $-20.7 \mathrm{mV}$. A substantial number of GDE nanoparticles was counted via NTA, at $2.05 \times 10^{13}$ particles $/ \mathrm{mL}$. Although differential centrifugation coupled to ultracentrifugation has been the most widely adopted method in this context, the impact of repeated ultracentrifugation steps on the recovery of exosome-like nanoparticle yields has not been fully assessed [12]. There is an opinion that repeated ultracentrifugation and freeze-drying steps may damage isolated vesicles, reducing their quality [40]. However, using sequential centrifugation coupled with a sucrose cushion method, uniform and reproducible GDEs with a high yield and purity were successfully isolated. Additionally, we investigated the stability and potential utility of freeze-dried GDEs by evaluating their protein content and total RNA concentration.

Ginseng has several beneficial properties in human health and has been used for thousands of years. As ginseng has various bioactive activities, even before the use of drugs, it has been considered as one of the most useful medicinal herbs for human health and longevity until today [41]. Particularly in Korea, the word "Panax" in the name of Asian ginseng (Panax ginseng Meyer) means "all healing," and explains the traditional belief that "ginseng can heal all aspects of the body." If certain components of ginseng can benefit the human body, these specific factors and characteristics must be utilized. The present method will have extensive applications in the field of extracellular vesicle research. Additionally, this method produces GDEs with high yield and purity, promoting their active use in the clinical field. Owing to the observed stability of freeze-dried GDEs at room temperature for up to 60 days, it shows that GDEs obtained through this simple method can be easily stored and have the potential for use at an appropriate time after mass production [42]. The protocol demonstrated in this study shows potential for the efficient isolation and mass production of unique GDEs from 
anywhere in the world. The GDEs obtained in this manner also show potential as a biologically active substance and a delivery vehicle for use in sustainable therapeutic strategies. Further studies are needed to determine which substances in GDE cargoes can cause specific therapeutic effects and which properties of GDEs can be used for therapeutic application and drug delivery.

\section{Conclusions}

In this study, we isolated GDEs with a less laborious, simple and reproducible way using a sucrose cushion ultracentrifugation. GDEs were verified with their physical, chemical characteristics and stability in various aspects including morphological analysis measured by DLS, TEM, and NTA and bioactive molecules. GDEs showed a homogeneous circular shape with a high purity and a high stability for 60 days after freeze-drying. The sucrose cushion ultracentrifugation is promising for applications in the research for plant-derived exosome-like nanoparticles. Besides, we suggest that GDEs are potential nanovesicles with exosome-like properties and conclude that GDEs could mediate intercellular communication as a drug or serve as an adjuvant and/or carrier of cytotoxic chemical drugs to target cells in drug delivery systems. We anticipate that GDEs with high reproducibility and stability can be applied in smart nanotechnology engineering for biomedical applications in the future.

\section{Materials and methods}

\subsection{Isolation of GDEs}

To obtain GDEs, the roots of 4-year-old P. ginseng were purchased from Geumsan Chaminsam (Geumsan, Korea). Fresh ginseng was washed with diethyl pyrocarbonate (DEPC) water three times and homogenized with $1 \times$ phosphate-buffered saline (PBS, Invitrogen, CA, USA) to make a slurry at room temperature $\left(20^{\circ} \mathrm{C}\right)$. The ground slurry was squeezed with a hand squeezer to remove impurities and collect the ginseng juice. The juice was then sequentially centrifuged at $2000 \times \mathrm{g}$ for $20 \mathrm{~min}$ and $10,000 \times \mathrm{g}$ for $60 \mathrm{~min}$. After centrifugation, the pellet was discarded and the supernatant was collected. Subsequently, the supernatant was passed through a sterilizing grade filter membrane (Acrodisc ${ }^{\oplus}$ Syringe Filters $0.2 \mu \mathrm{m}$, Pall Corporation, USA) to obtain nano-scale exosome-like nanoparticles. Sucrose cushions ( $27 \%$ and $68 \%$ sucrose) were layered on the bottom of a centrifuge tube, and the supernatant was gently transferred to the cushion. After ultracentrifugation at $100,000 \times \mathrm{g}$ for $90 \mathrm{~min}$, the extracellular vesicles between the clear sucrose cushion layers were collected. The total extracellular vesicles were then transferred to a gradient sucrose solution ( $8 \%, 30 \%, 45 \%$, and $60 \%$ ) and ultracentrifuged at $200,000 \times \mathrm{g}$ for another $90 \mathrm{~min}$. All centrifugation steps were performed at $4{ }^{\circ} \mathrm{C}$. According to the density of sucrose used, GDEs were isolated at concentrations between 8 and $30 \%(1.13 \sim 1.19 \mathrm{~g} / \mathrm{mL}$ concentration) The isolated GDEs were stored at $-80^{\circ} \mathrm{C}$ until use (Fig. 2).

\subsection{Dynamic light scattering (DLS)}

The size distribution and zeta potential of the GDEs were measured via dynamic light scattering (DLS) using a Zetasizer nano ZS Zen3600 (Malvern, UK). The GDEs were diluted 1:1000 in DEPC water to a total volume of $1 \mathrm{~mL}$. The diluted GDEs were then transferred to a cuvette $(12 \mathrm{~mm}$ square polystyrene cuvette, DTS0012, Malvern, UK) and added to a disposable cuvette for zeta potential measurement (disposable capillary cell, DTS1070, Malvern, UK). The measurements were performed at $37{ }^{\circ} \mathrm{C}$, and all experiments were run in triplicate.

\subsection{Nanoparticle tracking analysis (NTA)}

To quantify the GDEs, their sizes and number of particles were assessed using a NanoSight LM14 instrument (Malvern Instruments, Malvern, UK). The samples were diluted 1:1000 with $1 \times$ PBS, and $1 \mathrm{~mL}$ of the diluted sample was loaded into the laser chamber using a syringe. Five videos, approximately $60 \mathrm{~s}$ each in duration, were recorded with the camera setting at 13. Each sample was measured in triplicate.

\subsection{Transmission electron microscope (TEM) imaging}

The GDEs ( $10 \mu \mathrm{L}$ of $10 \mu \mathrm{g}$ GDEs) were spotted onto carbon-coated grids at room temperature for $10 \mathrm{~min}$. The carbon-coated grids were immediately stained with $2 \%$ phosphotungstic acid at room temperature for $30 \mathrm{~min}$. Nanoparticle imaging was conducted using a transmission electron microscope (TEM; JEM-F200, JEOL, Japan).

\subsection{Lyophilization of GDEs (Freeze drying)}

Fresh GDEs were stored in a deep freezer for $24 \mathrm{~h}$ as the primary drying step for low-temperature drying. The frozen GDEs were quickly transferred to a freeze-drying chamber and placed under vacuum to dehydrate the frozen samples. After $96 \mathrm{~h}$ in vacuum, freeze-dried GDEs were obtained. 


\subsection{Measurement of total RNA concentration}

Total RNA was extracted using TRIzol reagent according to the manufacturer's instructions (Invitrogen, Carsbad, CA, USA). Briefly, $0.1 \mathrm{~g} \mathrm{GDEs}$ were dissolved in $0.25 \mathrm{~mL} 1 \times \mathrm{PBS}$ and $0.75 \mathrm{~mL}$ TRIzol reagent was added to the GDE solution. To this, $0.25 \mathrm{~mL}$ of chloroform was added, thoroughly mixed, and incubated at $-20^{\circ} \mathrm{C}$ for $10 \mathrm{~min}$. The sample was then centrifuged at $12,000 \times \mathrm{g}$ for $15 \mathrm{~min}$, and the aqueous phase was carefully collected without disturbing the interphase, transferred into a new tube, and an equal volume of isopropanol was added. Afterwards, the sample was centrifuged at $12,000 \times \mathrm{g}$ for $10 \mathrm{~min}$. The supernatant was removed and the pellet was resuspended in $70 \%$ ethanol for washing, and the sample was centrifuged at $7500 \times \mathrm{g}$ for $5 \mathrm{~min}$. The supernatant was discarded, and the pellet at the bottom of the tube was dried for $10 \mathrm{~min}$. The pellet was resuspended in $50 \mu \mathrm{L}$ of DNase/RNase-free distilled water (Invitrogen). All centrifugation procedures were performed at $4{ }^{\circ} \mathrm{C}$. Total RNA was quantified by measuring the absorbance at $260 \mathrm{~nm}$ using a UV-Vis spectrophotometer (NanoDrop 2000, Thermo Scientific, USA).

\subsection{Measurement of protein concentration}

To quantify GDEs, total protein of GDEs was analyzed by Bradford protein assay. The isolated GDEs were placed in different containers and stored at room temperature. The protein concentration was quantified for 30,60 , and $90 \mathrm{~d}$.

\subsection{Statistical analysis}

In each experiment, triplicate experiments were performed for each condition or treatment group. Data were analyzed using IBM SPSS Statistics for Windows Version 26.0 (IBM Corp., Armonk, NY, USA) and are presented as means \pm standard deviations (SDs). The unpaired t-test and one-way ANOVA followed by multiple comparisons by Tukey's post-hoc analysis were used to assess differences between groups. Statistical significance was set at $P \leq 0.05$. The statistical significance of the data is indicated with asterisks: ${ }^{*}, p<0.05$, and ${ }^{* *}, p<0.001$.

Author contributions $\mathrm{Y}-\mathrm{HL}$ and JK wrote the main manuscript text and Y-HL and JK prepared Fig. 1-7. JW and IIKK advised on the composition of the manuscript based on their experience and knowledge, and YKK and other authors proceeded with data collection. All authors reviewed the manuscript.

Funding This work was supported by a National Research Foundation of Korea (NRF) grant funded by the Korean government (MSIT) (No. NRF-2020R1F1A1070072).

\section{Declarations}

Conflict of interests The authors declare that they have no competing interests.

Consent for publication The authors declare no potential conflicts of interest with respect to the research, authorship, or publication of this article.

Open Access This article is licensed under a Creative Commons Attribution 4.0 International License, which permits use, sharing, adaptation, distribution and reproduction in any medium or format, as long as you give appropriate credit to the original author(s) and the source, provide a link to the Creative Commons licence, and indicate if changes were made. The images or other third party material in this article are included in the article's Creative Commons licence, unless indicated otherwise in a credit line to the material. If material is not included in the article's Creative Commons licence and your intended use is not permitted by statutory regulation or exceeds the permitted use, you will need to obtain permission directly from the copyright holder. To view a copy of this licence, visit http://creativecommons. org/licenses/by/4.0/.

\section{References}

1. Li X, Mo N, Li Z (2020) Ginsenosides: potential therapeutic source for fibrosis-associated human diseases. J Ginseng Res 44:386398. https://doi.org/10.1016/j.jgr.2019.12.003

2. Ratan ZA et al (2021) Pharmacological potential of ginseng and its major component ginsenosides. J Ginseng Res 45:199-210. https://doi.org/10.1016/j.jgr.2020.02.004

3. Lee S, Rhee D-K $(2017)$ Effects of ginseng on stress-related depression, anxiety, and the hypothalamic-pituitary-adrenal axis. J Ginseng Res 41:589-594. https://doi.org/10.1016/j.jgr. 2017.01.010

4. Hou W, Wang Y, Zheng P, Cui R (2020) Effects of ginseng on neurological disorders. Front Cell Neurosci 14:55-55. https://doi. org/10.3389/fncel.2020.00055

5. Kim MK et al (2018) Antinociceptive and anti-inflammatory effects of ginsenoside Rf in a rat model of incisional pain. J Ginseng Res 42:183-191. https://doi.org/10.1016/j.jgr.2017.02.005

6. He F, Yu C, Liu T, Jia H (2020) Ginsenoside Rg1 as an effective regulator of mesenchymal stem cells. Front Pharmacol 10:15651565. https://doi.org/10.3389/fphar.2019.01565

7. Woith E, Fuhrmann G, Melzig MF (2019) Extracellular vesiclesconnecting kingdoms. Int J Mol Sci 20:5695. https://doi.org/ 10.3390/ijms20225695

8. Wang $Y$ et al (2020) Exosomes as mediators of cell-to-cell communication in thyroid disease. Int J Endocrinol. https://doi. org/10.1155/2020/4378345

9. Mentkowski KI, Snitzer JD, Rusnak S, Lang JK (2018) Therapeutic potential of engineered extracellular vesicles. AAPS J 20:50. https://doi.org/10.1208/s12248-018-0211-z

10. Fu S, Wang Y, Xia X, Zheng JC (2020) Exosome engineering: Current progress in cargo loading and targeted delivery. Nanolmpact 20:100261. https://doi.org/10.1016/j.impact. 2020.100261

11. Ha D, Yang N, Nadithe V (2016) Exosomes as therapeutic drug carriers and delivery vehicles across biological membranes: current perspectives and future challenges. Acta Pharm Sin B 6:287-296. https://doi.org/10.1016/j.apsb.2016.02.001 
12. Doyle LM, Wang MZ (2019) Overview of extracellular vesicles, their origin, composition, purpose, and methods for exosome isolation and analysis. Cells 8:727. https://doi.org/10.3390/ cells8070727

13. Fernandes M, Lopes I, Teixeira J, Botelho C, Gomes AC (2020) Exosome-like nanoparticles: a new type of nanocarrier. Curr Med Chem 27:3888-3905. https://doi.org/10.2174/09298 67326666190129142604

14. Perut F et al (2021) Strawberry-derived exosome-like nanoparticles prevent oxidative stress in human mesenchymal stromal cells. Biomolecules. https://doi.org/10.3390/biom11010087

15. Zhang M, Viennois E, Xu C, Merlin D (2016) Plant derived edible nanoparticles as a new therapeutic approach against diseases. Tissue Barriers 4:e1134415-e1134415. https://doi. org/10.1080/21688370.2015.1134415

16. Ju S et al (2013) Grape exosome-like nanoparticles induce intestinal stem cells and protect mice from DSS-induced colitis. Mol Ther 21:1345-1357. https://doi.org/10.1038/mt.2013. 64

17. Luan X et al (2017) Engineering exosomes as refined biological nanoplatforms for drug delivery. Acta Pharmacol Sin 38:754763. https://doi.org/10.1038/aps.2017.12

18. Ahuja A, Kim JH, Kim J-H, Yi Y-S, Cho JY (2018) Functional role of ginseng-derived compounds in cancer. J Ginseng Res 42:248-254. https://doi.org/10.1016/j.jgr.2017.04.009

19. Zhang $Y$ et al (2020) Exosome: a review of its classification, isolation techniques, storage, diagnostic and targeted therapy applications. Int J Nanomedicine 15:6917-6934. https://doi. org/10.2147/IJN.S264498

20. Wu Y, Deng W, Klinke DJ., 2nd. (2015) Exosomes: improved methods to characterize their morphology, RNA content, and surface protein biomarkers. Analyst 140, 6631-6642. https:// doi.org/10.1039/c5an00688k

21. Li Z et al (2018) Arrowtail RNA for ligand display on ginger exosome-like nanovesicles to systemic deliver siRNA for cancer suppression. Sci Rep 8:14644. https://doi.org/10.1038/ s41598-018-32953-7

22. Webber J, Clayton A (2013) How pure are your vesicles? J Extracell Vesicles. https://doi.org/10.3402/jev.v2i0.19861

23. Lobb RJ et al (2015) Optimized exosome isolation protocol for cell culture supernatant and human plasma. J Extracell Vesicles 4:27031-27031. https://doi.org/10.3402/jev.v4.27031

24. Patel GK et al (2019) Comparative analysis of exosome isolation methods using culture supernatant for optimum yield, purity and downstream applications. Sci Rep 9:5335. https:// doi.org/10.1038/s41598-019-41800-2

25. Lamparski HG et al (2002) Production and characterization of clinical grade exosomes derived from dendritic cells. J Immunol Methods 270:211-226. https://doi.org/10.1016/s00221759(02)00330-7

26. Van Deun J et al (2014) The impact of disparate isolation methods for extracellular vesicles on downstream RNA profiling. J Extracell Vesicles. https://doi.org/10.3402/jev.v3.24858

27. Konoshenko MY, Lekchnov EA, Vlassov AV, Laktionov PP (2018) Isolation of extracellular vesicles: general methodologies and latest trends. Biomed Res Int 2018:8545347. https://doi.org/ $10.1155 / 2018 / 8545347$

28. Lobb RJ et al (2015) Optimized exosome isolation protocol for cell culture supernatant and human plasma. J Extracell Vesicles 4:27031. https://doi.org/10.3402/jev.v4.27031
29. Gupta S et al (2018) An improvised one-step sucrose cushion ultracentrifugation method for exosome isolation from culture supernatants of mesenchymal stem cells. Stem Cell Res Ther 9:180. https://doi.org/10.1186/s13287-018-0923-0

30. Cao $M$ et al (2019) Ginseng-derived nanoparticles alter macrophage polarization to inhibit melanoma growth. J Immunother Cancer 7:326. https://doi.org/10.1186/ s40425-019-0817-4

31. Duong P, Chung A, Bouchareychas L, Raffai RL (2019) Cushioned-density gradient ultracentrifugation (C-DGUC) improves the isolation efficiency of extracellular vesicles. PLoS ONE 14:e0215324. https://doi.org/10.1371/journal.pone. 0215324

32. Fujita D et al (2018) Apple-derived nanoparticles modulate expression of organic-anion-transporting polypeptide (OATP) 2B1 in caco-2 cells. Mol Pharm 15:5772-5780. https://doi.org/ 10.1021/acs.molpharmaceut.8b00921

33. Komori $\mathrm{H}$ et al (2021) MicroRNAs in apple-derived nanoparticles modulate intestinal expression of organic anion-transporting peptide 2B1/SLCO2B1 in caco-2 cells. Drug Metab Dispos 49:803-809. https://doi.org/10.1124/dmd.121.000380

34. Mu J et al (2014) Interspecies communication between plant and mouse gut host cells through edible plant derived exosome-like nanoparticles. Mol Nutr Food Res 58:1561-1573. https://doi.org/10.1002/mnfr.201300729

35. Wang Q et al (2015) Grapefruit-derived nanovectors use an activated leukocyte trafficking pathway to deliver therapeutic agents to inflammatory tumor sites. Cancer Res 75:2520-2529. https://doi.org/10.1158/0008-5472.Can-14-3095

36. Suresh AP, Kalarikkal SP, Pullareddy B, Sundaram GM (2021) Low $\mathrm{pH}$-based method to increase the yield of plant-derived nanoparticles from fresh Ginger Rhizomes. ACS Omega 6:17635-17641. https://doi.org/10.1021/acsomega.1c02162

37. You JY, Kang SJ, Rhee WJ (2021) Isolation of cabbage exosomelike nanovesicles and investigation of their biological activities in human cells. Bioact Mater 6:4321-4332. https://doi.org/ 10.1016/j.bioactmat.2021.04.023

38. Zu M et al (2021) "Green" nanotherapeutics from tea leaves for orally targeted prevention and alleviation of colon diseases. Biomaterials 279:121178. https://doi.org/10.1016/j.biomateria Is.2021.121178

39. Zhuang $X$ et al (2015) Ginger-derived nanoparticles protect against alcohol-induced liver damage. J Extracell Vesicles 4:28713. https://doi.org/10.3402/jev.v4.28713

40. Burnouf T, Agrahari V, Agrahari V (2019) Extracellular vesicles as nanomedicine: hopes and hurdles in clinical translation. Int J Nanomedicine 14:8847-8859. https://doi.org/10.2147/ IJN.S225453

41. Yang Y, Ren C, Zhang Y, Wu X (2017) Ginseng: an nonnegligible natural remedy for healthy aging. Aging Dis 8:708-720. https://doi.org/10.14336/AD.2017.0707

42. Jeyaram A, Jay SM (2017) Preservation and storage stability of extracellular vesicles for therapeutic applications. AAPS J 20:1-1. https://doi.org/10.1208/s12248-017-0160-y

Publisher's Note Springer Nature remains neutral with regard to jurisdictional claims in published maps and institutional affiliations. 